Available online on 15.12.2019 at http://jddtonline.info
Open Access to Pharmaceutical and Medical Research
unrestricted non-commercial use, provided the original work is properly cited

Open $\odot$ Access

Research Article

\title{
Fabrication of drug eluting medical device for treating stenosis by 3D printing and dip coating using aspirin as a model drug
}

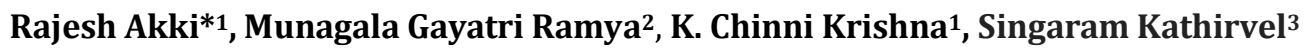 \\ ${ }^{1}$ Assistant Professor, Department of Pharmaceutics, Hindu college of Pharmacy, Amaravathi Road, Guntur-522002, Andhra Pradesh, India. \\ 2 University College of Pharmaceuical Sciences, Acharya Nahajuna University, Nagarjuna Nagar, Guntur, Andhra Pradesh, India \\ ${ }^{3}$ National College of Pharmacy, Manassery P.0, Mukkam, Kozhikode-673602, Kerala
}

\begin{abstract}
3D printing is a new innovative manufacturing method for fabrication of customized medical devices. The customized medical devices \& longlasting implantable devices.has increasing demand for addressing some critical cases in surgeries.

The main aim of this work was to explore the potential of 3D printing in Fabrication of medical devices and prosthetics. The characters of the polymers, the features of softwares were studied.

The study showed that drug loading into filament through hot melt extrusion and followed by 3D printing has many defects such as denaturing of drugs at higher printing temperatures.

The invention discloses the dip coating process after fabrication of a 3D printed polymer structure. The drug release depends up on the surface area of the device, coated polymer, concentration of drug and thickness of the coat.

The method for preparing the personalized drug eluting coronary stent / Bone wedges / Braces comprises the step that according to image data of coronary angiogram or volume rendered data from CT scans. The designing was done by adopting a QCA technique for measuring the diameter of a diseased coronary artery and reconstructing in a three-dimensional manner. According to indexes such as lesion vascular diameter, lesion length and lesion vascular pattern, a personalized coronary stent can be made for each patient in a customized manner and a stent most suitable for the lesion state of a patient can be prepared.
\end{abstract}

Keywords: 3D printing, manufacturing method, Fabrication of medical devices

Article Info: Received 12 Oct 2019; Review Completed 21 Nov 2019; Accepted 29 Nov 2019; $\quad$ Available online 15 Dec 2019

Cite this article as:

Akki R, Ramya MG, Krishna KC, Kathirvel S, Fabrication of drug eluting medical device for treating stenosis by 3D printing and dip coating using aspirin as a model drug , Journal of Drug Delivery and Therapeutics. 2019; 9(6-s):148-154 http://dx.doi.org/10.22270/jddt.v9i6-s.3767

*Address for Correspondence:

Rajesh Akki, Assistant Professor, Department of Pharmaceutics, Hindu college of Pharmacy, Amaravathi road, Guntur-522002, Andhra Pradesh, India.

\section{INTRODUCTION:}

Medical device means any instrument, apparatus, implant, machine, appliance, implant, in vitro reagent or calibrator, software, material or other similar or related articles, intended by the manufacturer to be used, alone or in combination, for human beings for one or more of the specific purposes like diagnosis, prevention, monitoring, treatment or alleviation of disease; diagnosis, monitoring, treatment, alleviation of or compensation for an injury; investigation, replacement, modification or support of the anatomy or of a physiological process; supporting or sustaining life; control of conception; disinfection of medical devices and providing information for medical purposes by means of in vitro examination of specimens derived from the human body and does not achieve its primary intended action in or on the human body by pharmacological, immunological or metabolic means, but may be assisted in its function by such means

There are many new innovative manufacturing like CNC laser cutting [2],Injection Molding [3], and 3D Printing [3][4][5][6].

3D Printing have more Precision and have more advantages compared to injection molding[7]. 3D printing is an additive manufacturing process which creates a physical object from a digital design, by laying down thin layers of material in the form of liquid or powdered polymers, metal or cement which is fused together [8]. 
However, 3D printing speeds are still too slow for mass production. For now, the technology is mainly reducing the lead time in the development of prototypes of parts and devices, and the tooling needed to make them. This is a huge benefit to smaller scale manufacturers because it reduces costs and time to market. Because 3D printing can create intricate and complex shapes using less material than subtractive manufacturing processes like milling[9].

\section{Literature Survey:}

While currently used primarily to manufacture prototypes and mockups, a number of promising applications exist in the production of replacement parts, dental crowns, and artificial limbs,bone wedges, implants as well as in bridge manufacturing. Antonio J. Guerra , Paula Cano Authors has worked to produce PCL/PLA composite stents using a novel 3D tubular printer based on Fused Deposition Modeling (FDM) ${ }^{[10]}$.

Antonio J. Guerra, Paula Cano proved that the 3D-printing process was highly suitable for producing composite stents[11]. Yang Lei, Xin Chen had manufactured a high efficient personalized stent by additive manufacturing (3D printing) has gained deal of attention and research in interventional and personalized medicine ${ }^{[12]}$.

FDA-approved 3D printed implants by Osseus Fusion systems that are patient-specific where the implant is designed with that specific patient's anatomy in mind.Degenerative Disc Disease, for example, affects more than 30 million people a year, and tends to occur as bone density decreases in a person's later years, drying out and shrinking their discs, which in turn tightens the space around the nerves and spinal cord. In an attempt to prevent pains occurring, surgeons will look to remove the worn disc and replace it with a titanium interbody[13]

It is necessary to have proper understanding of medical device safety, risk involved, the degree of invasiveness, duration of contact, the body system affected, and local versus systemic effects. Pharmacist should be actively involved in the regulation of effectiveness and performance of medical device. Clinical effectiveness is a good indicator of device performance, which is closely linked to safety. Pharmacists should be involved in the documentary of standards containing technical specifications or other precise criteria to be used consistently as rules, guidelines or definitions of characteristics, to ensure that materials, products, process and services are fit for their purpose. One has to ensure that the prescriptive, design, performance, and management specifications meet the standards [1].

XIAOHUA LIU1 and PETER X. MA has described two major disadvantages of Metals and ceramics for tissue engineering applications: they are lack of degradability in a biological environment, and their process ability is very limited. In contrast, polymers have great design flexibility because the composition and structure can be tailored to the specific needs [14].

Bret D. Ulery et al.[15], A. Ashwin Kumar et al.[16], AnezkaLengalova et al.[17]Shenguo Wang et al.[18]Mark Chasin\& Robert Langer[19] , had described the use of biodegradable polymers in Tissue engineering, Implantable medical devices, Prosthetics \& other medical applications.MihaiRusu et al.[20], Bret D. Ulery et al.[21], had described the uses of Polycaprolactone in medical applications and A. Anand et al.[22], M. Kammerer et al.[23] had used Polycaprolactone for fabrication of implantable medical devices.

Jenny Hollander et al [24] fabricate drug-containing T-shaped prototypes of intrauterine system (IUS) with the drug incorporated within the entire backbone of the medical device using 3-dimensional (3D) printing technique, based on fused deposition modeling

\section{Software Methods}

\section{DICOM format ${ }^{[25]}$}

A DICOM file is an image saved in the Digital Imaging and Communications in Medicine (DICOM) format. The DICOM format was developed by the NEMA (National Electrical Manufacturers Association). It was designed for the exchanging and viewing of medical images, such as CT scans, MRIs, and ultrasound images. It contains an image from a medical scan, such as an ultrasound or MRI. DICOM files may also include identification data for patients so that the image is linked to a specific individual.

There are various softwares for viewing dicom files like MICRO DICOM[26] and MEDDREAM DICOM[27] viewer. The diameters of vessels and measurements can be taken by measurement and various tools in the softwares.

Download free software from

http://www.microdicom.com/downloads.html.

We can also download free sample dicom file from various DICOM databases like osirix.

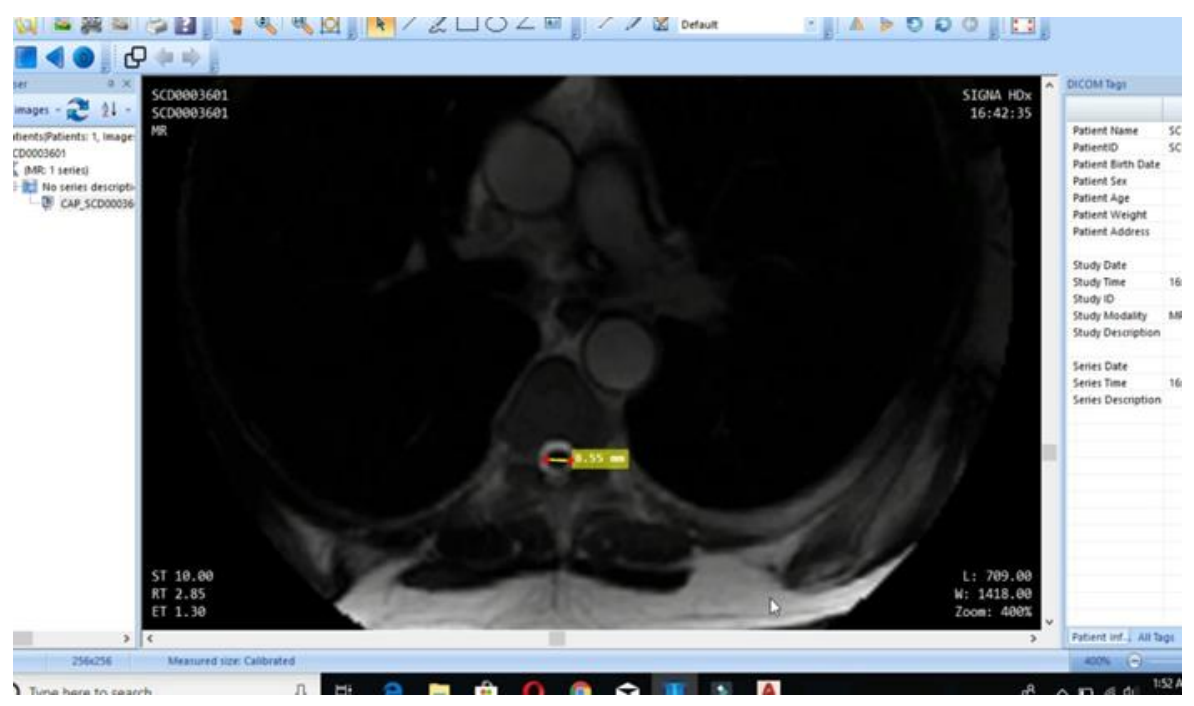

Fig.1 Microdicomdicom viewer software 


\section{Slicer Software[28]}

The slicer software is used to obtain the data from a CT-scan for visualizing and analysis of tissues and to obtain data for designing customized medical devices like Bone wedges, implants, prosthetics etc.slicer is a completely free open- source software for visualization and image analysis. this software has wide set of features like converting DICOM files to STL files and in which ever format we need. The features and capabilities of Slicer software was explained in slicer wiki. The slicer software can be installed directly from slicer software official website.

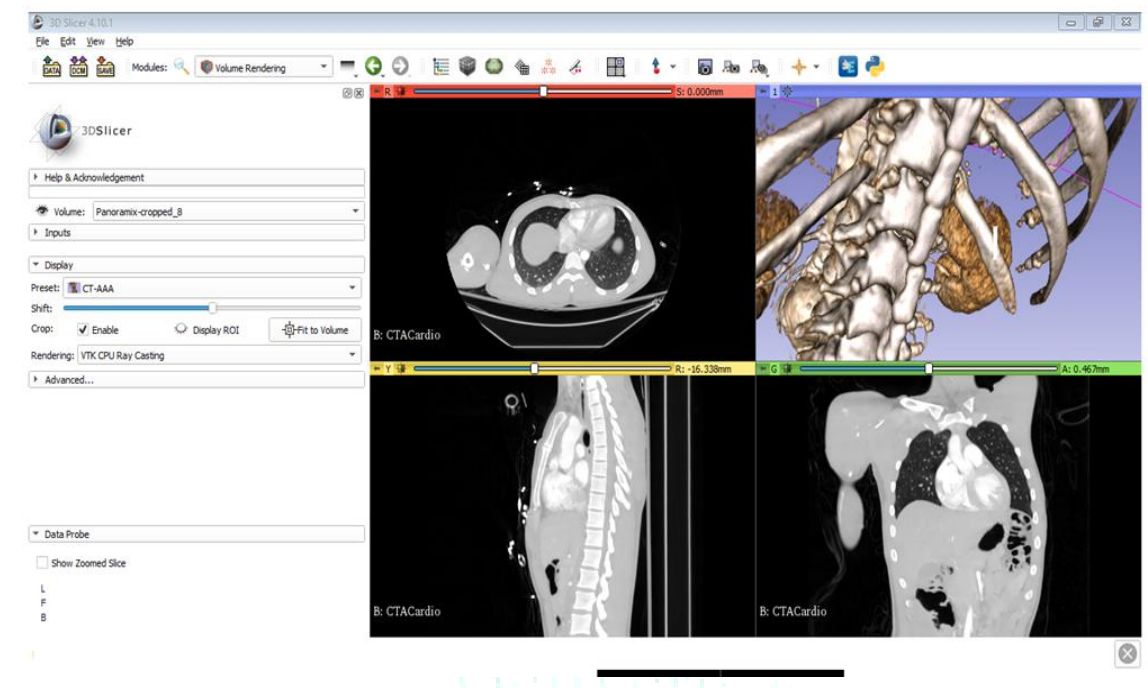

Fig2.3D Slicer software.

\section{Computer Aided Design \& Drafting}

The design was patient-specific where the implant is designed with that specific patient's anatomy in mind.[29].the design was done on CADD softwares like Autodesk AUTOCAD or SOLIDWORKS software

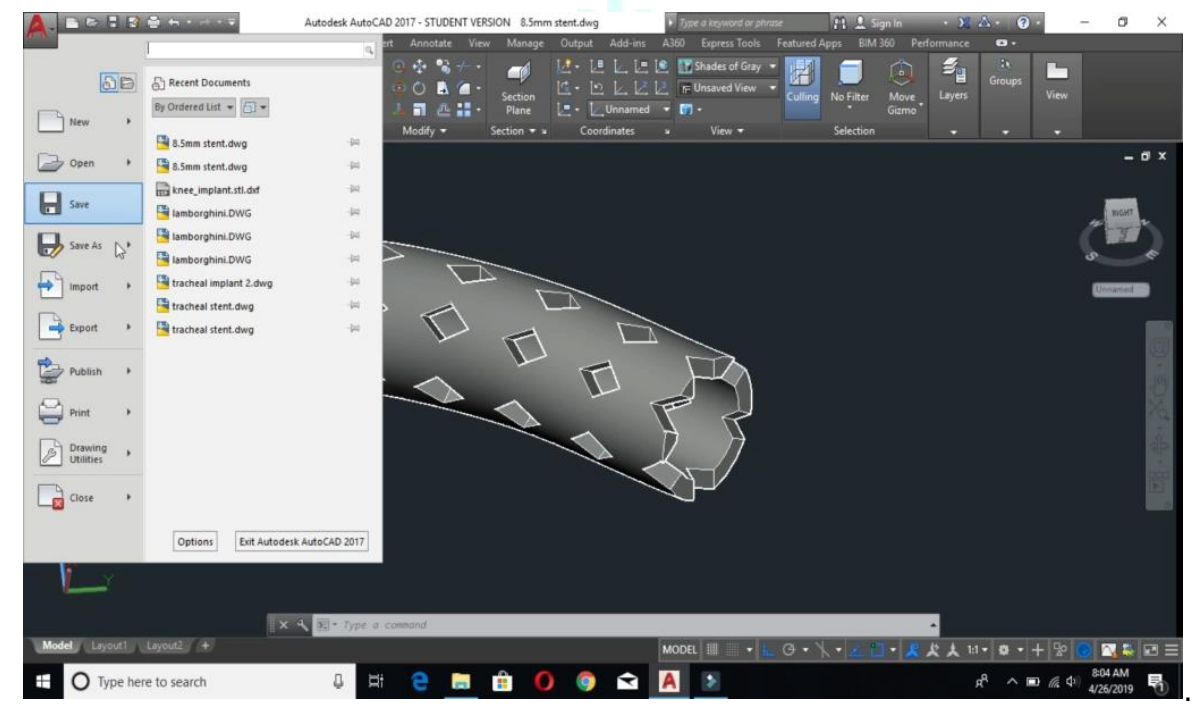

Fig3. Designing of Device in CADD software.

\section{STL file format.[29]}

STL (an abbreviation of "stereolithography") is a file format native to the stereolithography CAD software created by 3D Systems. STL has several backronyms such as "Standard Triangle Language" and "Standard Tessellation Language". This file format is supported by many other software packages; it is widely used for rapid prototyping, 3D printing and computer-aided manufacturing.STL files describe only the surface geometry of a three-dimensional object without any representation of color, texture or other common CAD model attributes.

\section{D Printing software [30]}

The slicer, also called slicing software, is computer software used in the majority of 3D printing processes for the conversion of a $3 \mathrm{D}$ object model to specific instructions for the printer. In particular, the conversion from a model in STL format to printer commands in g-code format in fused filament fabrication and other similar processes. 


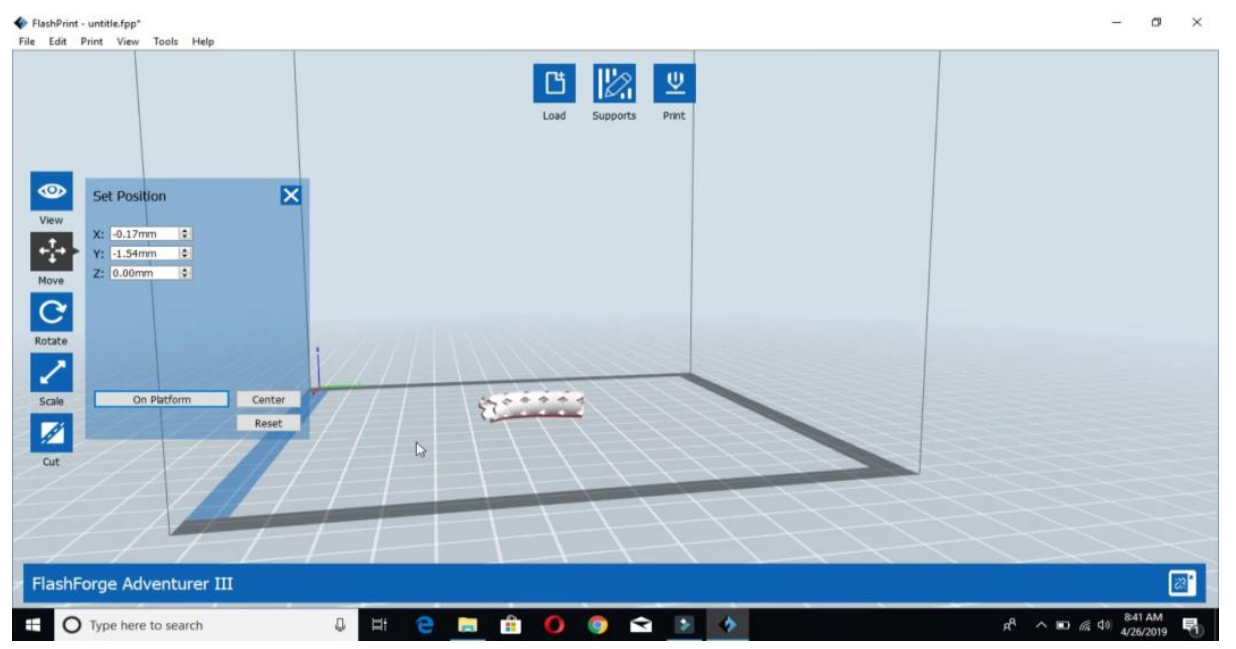

Fig4. FLASHFORGE FLASHPRINT slicer software.

\section{Fused Deposition Modelling (3D Printing) [31]}

The most common 3D printing technology is called the Fused Deposition Modelling (FDM) which utilises thermoplastic filaments as a starting material, then extrudes the material in sequential layers above its melting temperature to create a 3D object. . Fused deposition modeling (FDM) is a promising 3D printing technique for the fabrication of personalized drug dosage forms and patient-specific implants[32].

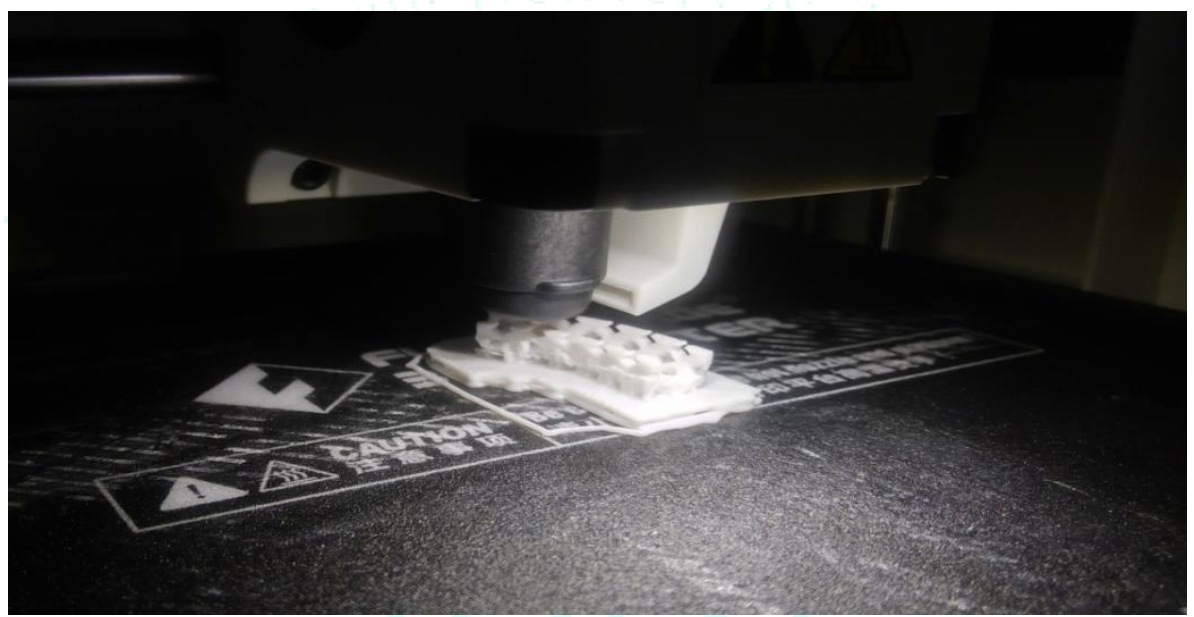

Fig 5.3D Printing of The device prototype.

\section{MATERIALS AND METHODS}

\section{Materials}

Polylactic acid filaments $1.75 \mathrm{~mm}$ was used for fabrication of the core structure of the device and Polycaprolactone was used in coating process. Aspirin was selected as a model drug \& Ethyl acetate was selected as solvent for dip coating The Polymer used in coating was polycaprolactone and the polymer used in fabricating the basic structure was polylactic acid. The solvent ethyl acetate was selected as the core structure would not be soluble and the polymer used in coating process must be soluble.

\section{Methods}

Zhou Yujie et al., in his patent [32] had explained The method for preparing the personalized drug eluting coronary stent comprises the step that according to image data of coronary angiogram, adopting a QCA technique for measuring the diameter of a diseased coronary artery and reconstructing in a three-dimensional manner.

According to indexes such as lesion vascular diameter, lesion length and lesion vascular pattern, a personalized coronary stent can be made for each patient in a customized manner and a stent most suitable for the lesion state of a patient can be prepared. The most suitable personalized bionic drug eluting coronary stent can be customized for the patient according to diseased coronary arteries of different states, requirements of different lesions can be met, customization of coronary stents can be achieved, and complications such as vascular injury, thrombus and coronary arterial dissection caused by mismatching of the diameter of the stent and that of the blood vessel can be reduced.

Jenny Hollander et al [24] fabricate drug-containing T-shaped prototypes of intrauterine system (IUS) with the drug incorporated within the entire backbone of the medical device using 3-dimensional (3D) printing technique, based on fused deposition modeling. According to Antonio J. Guerra et al.,[10] Polycaprolactone has less accuracy and less printability during complex structure fabrication. The temperature during $3 \mathrm{D}$ printing can damage the Thermolabile drugs during 3D printing process or Hot melt extrusion of filaments.

The Present study discloses the dip coating process ${ }^{[3]}$ after fabrication of a 3D printed polymer structure. The drug release depends up on various factors likecrystallinity of drug surface area of the device, coated polymer, 
concentration of drug and thickness of the coat.Three different concentrations of Aspirin in the polymer, namely $5 \%, 10 \%$, and $20 \%$, were selected for the coating process.

Polylactic acid filaments $1.75 \mathrm{~mm}$ was used for fabrication of the core structure of the device and Polycaprolactone\& aspirin was mixed in different concentrations namely $5 \%$, $10 \%$, and $20 \%$. Ethyl acetate was selected as solvent for dip coating of printed structures. The solvent ethyl acetate was selected as the core structure would not be soluble and the polymer used in coating process must be soluble.

\section{Rheology Measurement ${ }^{[24] \text {. }}$}

Rheology measurements were carried out to determine the viscosity of the polymer solutions for dip coating. The experiments were conducted with a rotational rheometer equipped with 2 parallel plates of $15 \mathrm{~mm}$ in diameter. $0.6 \mathrm{~g}$ of the sample was placed on the lower plate and the upper plate was lowered until there was $1 \mathrm{~mm}$ gap between the plates, and the sample was sheared between the plates. The viscosity was calculated as the ratio between the applied shear stress and the shear rate.

\section{In Vitro Drug Release [34]}

The drug release experiments were conducted with coated devices, containing different amounts of drug loading. Each sample was exactly weighed and placed in a compression spring made of stainless steel that was attached to the metal rod connected to the cap of a $250-\mathrm{mL}$ bottle. This assembly was needed to prevent the samples from lying on the bottom of the release vessel as the density of PCL is higher than the density of the release medium and is equal to $1.1 \mathrm{~g} / \mathrm{cm}^{3}$. Each sample was immersed into $200 \mathrm{~mL}$ of $0.9 \%$ sodium chloride. The bottles were placed on a shaking bath at $37.0 \pm$ $0.2 \mathrm{C}$ with shaking speed of $100 \mathrm{rpm}$ for 30 days. The release media was exchanged when needed to maintain sink conditions. Samples were taken at defined time points, and the concentration of indomethacin in the release medium was measured spectrophotometrically at $265 \mathrm{~nm}$.

\section{Matrix Degradation During In Vitro Release Tests}

To determine the mass loss of the polymer, the samples after the dissolution tests were dried at room temperature overnight and then weighed. The mass loss calculations were derived from the actual drug polymer ratio, obtained from drug content analysis. The released indomethacin percentage and polymer mass loss were calculated as follows:
Drug release percent $(\%)=[$ Weight of drug released $/$ Total actual weight of drug] $100 \%$

Mass loss of the polymer $(\%)=[($ The initial weight of sample - the final weight of sample - the weight drug released)/ Total actual weight of the polymer] x $100 \%$

\section{X-Ray Diffraction}

The degree of crystallinity of raw materials, their binary mixture (referred to the mixture of drug and polymer components prepared in the mortar), and HME filaments were studied with the help of an X-ray diffractometerXRD scans were performed in $\theta / 2 \theta$ Bragge Brentano geometry using CuKa radiation $(\lambda=1.54 \AA)$ with a voltage of $40 \mathrm{kV}$ and a current of $50 \mathrm{~mA}$, using a PIXcel 1D detector. [24] the samples were prepared by coating the polymer coating solution on a prototype that can fit in a sample holder.

\section{Fourier Transform Infrared Spectroscopy (FT-IR) [35]}

Structural characterization of the Polymer \& the drug and interactions between them were carried out by Fourier Transform Infrared Spectroscopy (FT-IR) using a Bruker spectrometer (Dept of Analysis,Hindu college of Pharmacy,AP). The spectra were recorded at ambient temperature between 400 and $4000 \mathrm{~cm}^{-1}$.

\section{Differential Scanning Calorimetry}

The differential scanning calorimetry is a tool used to measure the temperature and energy variation involved in the phase transitions, which reflects the degree of crystallinity and stability of the solid state of pharmaceutical compounds. The peak size and shape of the DSC curves are useful in determining the crystallinity of the drug and the carrier. In order toanalyze the association of aspirin with PCL,

\section{RESULTS}

\section{In Vitro Drug Release}

The in vitro drug release data for the PCL samples were fitted by zero-order, first-order, Higuchi, and Korsmeyere Peppas models The best fit was obtained with the zero order model ( $\mathrm{R}^{2}>0.99$ values for all samples), indicating that the diffusion is the predominant release mechanism of the drug from PCL polymer, regardless of the geometry of the samples and the amount of drug loading.

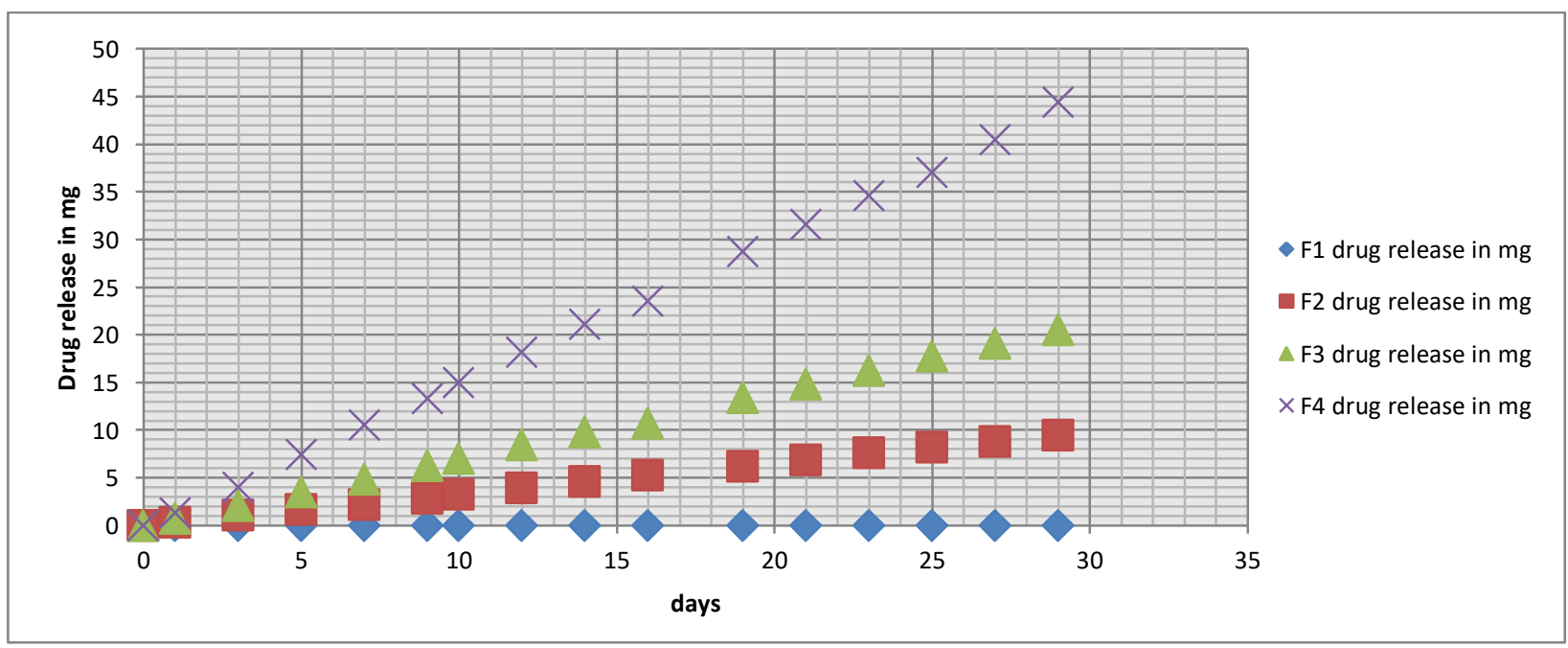

Fig 6.drug release vs time 
Table 1: Drug release kinetics of formulations F1,F2,F3.

\begin{tabular}{|c|c|c|c|c|c|}
\hline \multirow[t]{2}{*}{ Formulation } & \multirow[t]{2}{*}{$\begin{array}{l}\text { Zero order } \mathrm{R}^{2} \\
\mathrm{Mt} / \mathrm{M} \infty=\mathrm{Q}_{0}+\mathrm{k}_{0} \mathrm{t}\end{array}$} & \multirow[t]{2}{*}{$\begin{array}{l}\text { First order } \mathrm{R}^{2} \\
\log \mathrm{C}=\log \mathrm{C}_{0}-\mathrm{k}_{\mathrm{t}} / 2.303\end{array}$} & \multirow[t]{2}{*}{$\begin{array}{l}\text { Higuchi } \mathrm{R}^{2} \\
\mathrm{Mt} / \mathrm{M} \infty=\mathrm{k}_{\mathrm{H}} \cdot \mathrm{t}^{1 / 2}\end{array}$} & \multicolumn{2}{|c|}{$\begin{array}{l}\text { Kormeyers-Peppas } \\
\mathrm{Mt} / \mathrm{M} \infty=\mathrm{kt}^{\mathrm{n}}\end{array}$} \\
\hline & & & & $\mathrm{R}^{2}$ & release exponent $n$ \\
\hline F1 & NA & NA & NA & $\mathrm{NA}$ & NA \\
\hline F2 & 0.997 & 0.9767 & 0.8567 & 0.9058 & 1.056 \\
\hline F3 & 0.995 & 0.9623 & 0.8539 & 0.9162 & 0.997 \\
\hline $\mathrm{F} 4$ & 0.995 & 0.9536 & 0.8539 & 0.9036 & 1.004 \\
\hline
\end{tabular}

FTIR

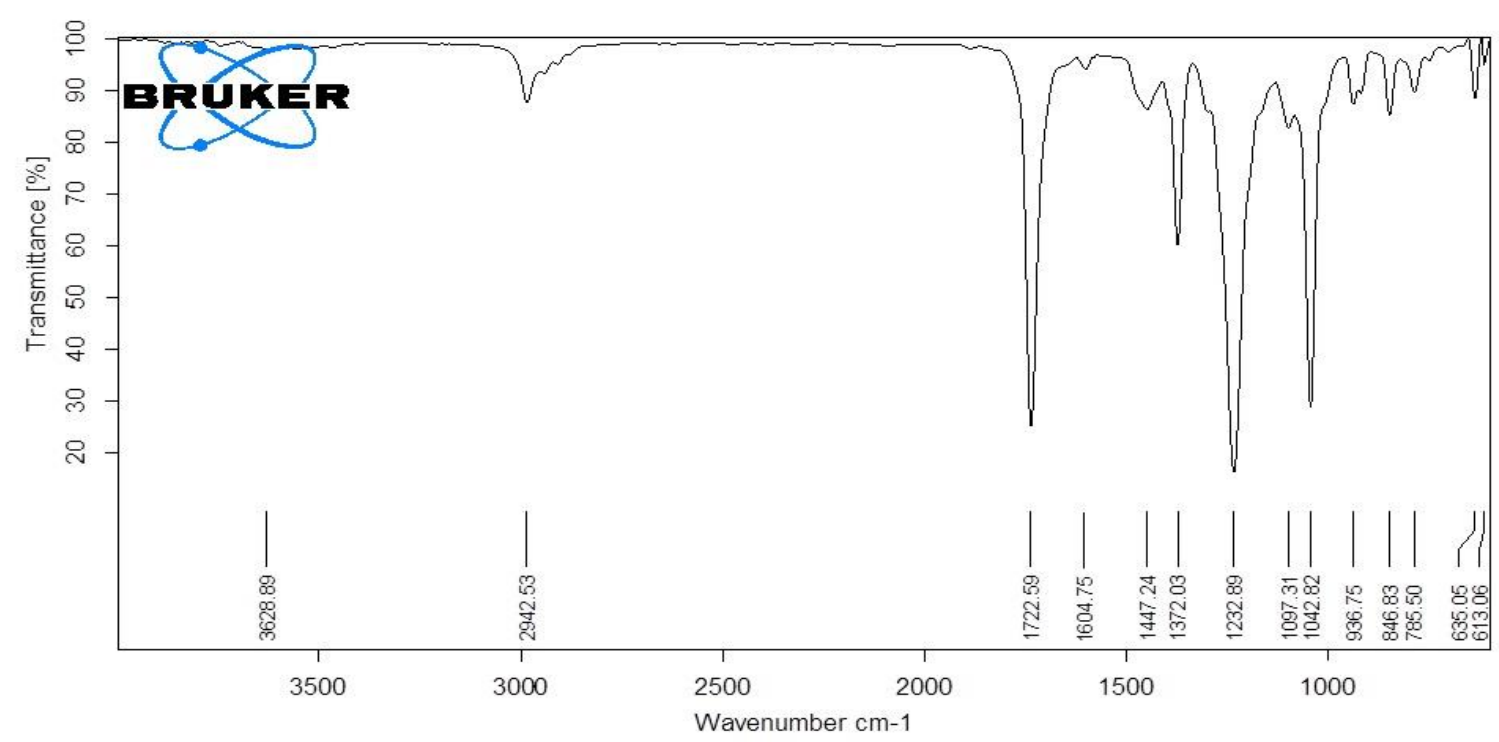

The peak at $1605 \mathrm{~cm}^{-1}$ is due to the benzene ring, whereas the absorption peak at $1680 \mathrm{~cm}^{-1}$ is due to the presence of carbonyl group in aspirin. These absorption peaks corresponded to the characteristic peaks of aspirin as described by $\mathrm{Li}$ et al (1991)[36]. The characteristic absorption bands at 2942 and $2865 \mathrm{~cm}^{-1}$ are related to C-H stretching vibration of hydrocarbon of PCL. The band at $1721 \mathrm{~cm}^{-1}$ is ascribed to the stretching vibration of carbonyl groups $(\mathrm{C}=0)$ of PCL[37].

\section{Matrix Degradation During In Vitro Release Test:}

The degradation of PCL is a 2-phase process with an initial molecular weight loss due to chain scission without a significant weight loss. When the loss of molecular weight is up to 5000, the mass loss begins due to the diffusion of small polymeric fragments out of the matrix[38].the mass loss of polymer was nearly $52 \%, 60 \%, 65 \%, 69 \%$ for $\mathrm{F} 1$ F4 formulations. The polymer degradation was very slow that it took over a month to degrade a thin coat of polymer.

\section{Differential Scanning Calorimetry;}

DSC analysis was performed on aspirin, PCL, and the aspirin-PCL mixture. The results of the DSC test confirmed there was no association of aspirin and PCL in the mixture as both peaks representing aspirin and PCL does not changed positions, while aspirin showed a sharp endothermic peak at $142.6^{\circ} \mathrm{C}$.

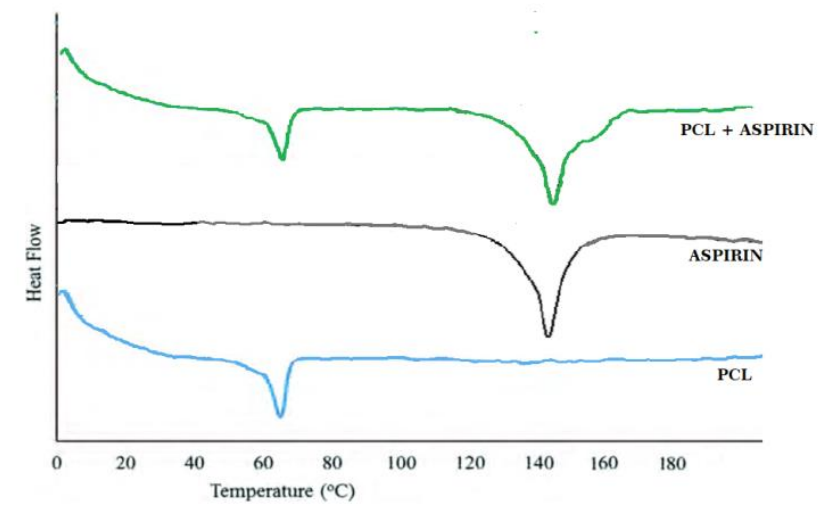

\section{CONCLUSION:}

The new innovative methods like injection moulding, CNC laser cutting and 3D printing has wide applications and has been employed in the manufacturing of medical Devices And implantable devices. 3D Printing is the process that can fabricate even tough and high complex designs.The methodology and software methods has been studied. the polymers used in fabrication has several advantages and their biocompatibility offers quality goal. Dip coating is used for coating a material used in sustain release mechanism The percentage of the dissolved drug was higher in the products with lower drug content. In vitro study reveals that diffusion was the predominant 
mechanism of the drug release from the coat insignificant influence by PCL biodegradation. Polycaprolactone had eroded and degraded very slowly that the loss is nearly 50 $60 \%$ in a month. Further studies will be performed to develop a design space for all other factors live coat surface area, polymers mixture ratios, polymer grades etc.

\section{REFERENCES}

1. Anuja R. Shah and R. K. Goyal, Current Status of the Regulation for Medical Devices, Indian J Pharm Sci. 2008 Nov-Dec; 70(6): 695-700.

2. Bogusz Stępak, Arkadiusz J. Antończak, and Krzysztof M. Abramski, Optimization of femtosecond laser cutting of a biodegradable polymer for medical devices manufacturing ,Photonics letters of poland, Vol. 8 (4), 116-118 (2016)

3. Sebastian Białasz1, and Tomasz Klepka, Simulation of the medical syringe injection moulding process ,MAETC web of conferences 252,05016(2019)

4. Yang Lei, Xin Chen, Lei Zhang, Lei Li, Shuangzhu Kang, Chengjin Wang and Wei Sun. Additive Manufacturing in Vascular Stent Fabrication

MATEC Web of Conferences 253, 03003 (2019)

5. Antonio J. Guerra, Paula Cano , Marc Rabionet, Teresa Puig and Joaquim Ciurana, Article 3D-Printed PCL/PLA Composite Stents: Towards a New Solution to Cardiovascular Problems, Materials 2018, 11, 1679

6. CN105877881A 2016-08-24 CN105877881A Application

7. Kathryn, Injection Molding vs. 3D Printing

8. www.investopedia.com/terms/1/3d-printing.asp

9. Robert Breitbach Consulting, Headhunter Printing Industry, , www.breitbach.com/h/headhunter-printing.htm

10. Barry Berman ,3-D printing: The new industrial revolution,

11. Antonio J. Guerra , Paula Cano , Marc Rabionet, Teresa Puig and Joaquim Ciurana 3D-Printed PCL/PLA Composite Stents: Towards a New Solution to Cardiovascular Problems

12. Yang Lei, Xin Chen, Lei Zhang, Lei Li, Shuangzhu Kang, , Chengjin Wangand Wei Sun Additive Manufacturing in Vascular Stent Fabrication

13. Sam Davies, FDA-approved 3D printed implants just the start as Osseus prepares to go international "We are trying to ride the wave of $3 \mathrm{D}$ printing.

14. Xiaohualiu and Peter x. ma, Polymeric Scaffolds for Bone Tissue Engineering, Annals of Biomedical Engineering, Vol. 32, No. 3, March 2004 (C2004) pp. 477-486.

15. Bret D. Ulery, Lakshmi S. Nair, Cato T. Laurencin. Biomedical Applications of Biodegradable Polymers, journal of polymer science part b: polymer physics 2011, 49, 832-864

16. A. Ashwin Kumar, Karthick. K, and K. P. Arumugam, Biodegradable Polymers and Its Applications International Journal of Bioscience, Biochemistry and Bioinformatics, Vol. 1, No. 3, September 2011

17. Anezka Lengalova, Alenka Vesel, Yakai Feng, and Vitor Sencadas Biodegradable Polymers for Medical Applications Hindawi Publishing Corporation International Journal of Polymer Science Volume 2016,

18. Shenguo Wang, Jianzhong Bei Biodegradable Polylactonefamily Polymers and Their Applications in Medical Field 8th arab international conference on polymer science \& technology 27 - 30 November 2005, Cairo-Sharm El-Shiekh, EGYPT

19. Mark Chasin, Robert Langer, Textbook of Biodegradable Polymers as Drug Delivery Systems

20. Gregory I. Peterson, Andrey V. Dobrynin, and Matthew L. Becker Biodegradable Shape Memory Polymers in Medicine Adv. Healthcare Mater. 2017, 1700694

21. Mihai Rusu, Mihaela Ursu, Daniela Rusu. Poly(vinyl chloride) and Poly(e-caprolactone) Blends for Medical Use. Journal of Thermoplastic Composite Materials, SAGE Publications (UK and US), 2006, 19 (2), pp.173-190.

22. Bret D. Ulery, Lakshmi S. Nair, and Cato T. Laurencin, Biomedical Applications of Biodegradable Polymers, J Polym Sci B Polym Phys. 2011 Jun 15; 49(12): 832-864.

23. A. ANAND, R. PUNDIR, C. S. PANDIAN, S. SARAF AND H. GUPTA Cefoperazone Sodium Impregnated Polycaprolactone Composite Implant for Osteomyelitis Indian J. Pharm. Sci., 2009, 71 (4): 377-381

24. Jenny Hollander , Natalja Genina , HarriJukarainen Mohammad Khajeheian, Ari Rosling , Ermei Makil , Niklas Sandler ,Three-Dimensional Printed PCL-Based Implantable Prototypes of Medical Devices for Controlled Drug Delivery

25. Fileinfo.com https://fileinfo.com/extension/dicom

26. www.microdicom.com/

27. MedDream DICOM Viewer - Softneta www.softneta.com/meddream.html

28. https://www.slicer.org/wiki/Documentation/4.10/FAQ

29. Evans, Brian. Practical 3D Printers: The Science and Art of 3D Printing. apress. ISBN 978-1-4302-4393-9.

30. BEGINNER'S GUIDE TO 3D PRINTING ,VERSION 0.1 ,THINK3D TEAM

31. Feuerbach T, Kock S, Thommes M Characterization of fused deposition modeling 3D printers for pharmaceutical and medical applications.

Pharm Dev Technol. 2018 Dec; 23(10):1136-1145

32. CA2276096C

33. Margarethe Richter, Christian Schneider ,Thermo Fisher Scientific ,Low-Temperature Hot-Melt Extrusion of Acetaminophen with EVA, 5781 Van Allen Way, Carlsbad, CA 92008, thermofisher.com

34. Cheng $\mathrm{L}, \mathrm{Guo} \mathrm{S}, \mathrm{Wu} \mathrm{W}$. Characterization and in vitro release of praziquantel from poly( $\varepsilon$-caprolactone) implants. Int J Pharm. 2009; 377:112-119.

35. Aspirin-loaded $\mathrm{P}(3 \mathrm{HO}) / \mathrm{P}(3 \mathrm{HB})$ blend films: Potential materials for biodegradable drug-eluting stents Patrick M. Aja , Onu Patience , Antoinette N. C. Okaka, Udu A. Ibiam Bioinspired, Biomimetic and Nanobiomaterials Volume 2 Issue BBN3

36. M. Shoja, K. Shameli, M. B. Ahmad , Z. Zakaria Preparation and characterization of poly (Ecaprolactone)/tio 2 microcomposite,

Digest Journal of Nanomaterials and Biostructures 2015; 10(2):471 - 477

37. Sinha VR, Bansal K, Kaushik R, Kumria R, Trehan A. Poly- $\varepsilon$ caprolactone microspheres and nanosperes: an overview. Int J Pharm. 2004; 278:1-23. 